\title{
P 232 END OF LIFE CARE IN NEURODEGENERATIVE CONDITIONS: EVALUATION OF A SPECIALIST NEUROLOGY PALLIATIVE CARE SERVICE
}

Jamilla Hussain, ${ }^{1}$ Debi Adams, ${ }^{2}$ Colin Campbell ${ }^{2} .{ }^{1}$ Leeds Teaching Hospitals NHS Trust, Leeds, UK; ${ }^{2}$ St. Catherine's Hospice, Scarborough

\subsection{6/bmjspcare-2014-000654.273}

Background The last year of life for people with advanced neurodegenerative conditions (ANC) is often unsatisfactory, characterised by multiple unplanned hospital admissions. Despite this few patients with ANC access palliative care services, with limited evidence of effective service models and when such individuals should be referred to palliative care.

Aims 1. To evaluate the Scarborough Palliative Neurology Service (PNS) model, led by specialist palliative neurology nurses, against the National End of Life Care Programme (NEoLCP) ANC recommendations. 2. To evaluate the validity of the seven NEOLCP triggers for palliative care referral.

Method Retrospective observational study of 62 consecutive patients under a PNS. Principle component analysis (PCA) was performed to examine the relationship between triggers and survival.

Results All patients had a key-worker who coordinated care. Capacity was assessed in $95 \%$ of cases, for who advance care planning and best interest decisions were documented in $95 \%$. The terminal-phase was recognised in $72.6 \%$ and $100 \%$ of carers were offered bereavement support.

The median follow-up under the PNS was 336 days. The mean number of hospital admissions was 0.9 in the last year of life (compared to 3.5 nationally across diagnoses). Pneumonia was the cause of death in $56.5 \%$. Thirty-one percent of patients died at home (nationally 14\% of ANC patients die at home), $26 \%$ in hospital (46\% nationally) and $24 \%$ in hospices $(0.03 \%$ nationally).

Frequency of triggers increased as patients approached death. PCA found 4 components in combination explained $76.8 \%$ of the variance in survival. These represented rapid physical decline; significant complex symptoms-including pain; infection with cognitive impairment; and risk of aspiration.

Conclusion A model of service provision investing in specialist palliative neurology nurses is an effective model in delivering palliative care to this vulnerable patient group. The triggers for referral require further validation, although the derived PCA components do have face-validity. 